\title{
Hedgehogs (Erinaceidae, Lipotyphla) from the Miocene of Pakistan, with description of a new species of Galerix
}

\section{Citation}

Zijlstra, Jelle, and Lawrence J. Flynn. 2015. Hedgehogs (Erinaceidae, Lipotyphla) from the Miocene of Pakistan, with description of a new species of Galerix." Palaeobio Palaeoenv 95, no. 3: 477-495. doi:10.1007/s12549-015-0190-3.

\section{Published Version}

10.1007/s12549-015-0190-3

\section{Permanent link}

http://nrs.harvard.edu/urn-3:HUL.InstRepos:26507533

\section{Terms of Use}

This article was downloaded from Harvard University's DASH repository, and is made available under the terms and conditions applicable to Other Posted Material, as set forth at http:// nrs.harvard.edu/urn-3:HUL.InstRepos:dash.current.terms-of-use\#LAA

\section{Share Your Story}

The Harvard community has made this article openly available.

Please share how this access benefits you. Submit a story.

\section{Accessibility}




\section{Electronic Supplement 1: Specimens examined}

This appendix lists all identifiable erinaceid specimens examined for this study. Age estimates from magnetostratigraphic information for Potwar and Zinda Pir localities follow locality numbers in parentheses. The two columns to the right are measurements in millimeters. Notation like "m1/2" means that the specimen is either an $\mathrm{m} 1$ or an $\mathrm{m} 2$, but could not be differentiated. For tooth-bearing jaws, we list the specimen itself under its main number, then any teeth inside the jaw under the number followed by a single-letter suffix.

\begin{tabular}{|c|c|c|c|c|}
\hline Specimen \# & Locality (Ma) & Element & Length & Widt \\
\hline \multicolumn{5}{|c|}{ Erinaceidae, gen. et sp. indet. } \\
\hline H-GSP 81.14a-4293 & Seh $81.14 \mathrm{a}$ & $\mathrm{m} 2$ & 2.37 & 1.28 \\
\hline YGSP 24485 & Y634 (12.3) & $\mathrm{m} 3$ & 1.4 & 0.53 \\
\hline YGSP 24499 & Y668 (13.4) & $\mathrm{m} 1$ & 2.25 & 1.16 \\
\hline \multicolumn{5}{|c|}{ Erinaceinae, gen. et sp. indet. } \\
\hline CH BS 403 & $\mathrm{CH}$ BS & $\mathrm{p} 4$ & 1.51 & 1.43 \\
\hline UJZ17 & Seh 81.14 & $\mathrm{~m} 1 / 2$ & $\mathrm{n} / \mathrm{a}$ & 2.17 \\
\hline YGSP 34899 & Y691 (13.1) & $\mathrm{m} 3$ & 1.63 & 1.21 \\
\hline YGSP 39462 & Y931 (7.3) & $\mathrm{m} 3$ & 1.28 & 1.05 \\
\hline YGSP 52204 & Z122 (18.9) & M1 & $\mathrm{n} / \mathrm{a}$ & $\mathrm{n} / \mathrm{a}$ \\
\hline
\end{tabular}

Galerix cf. rutlandae

\begin{tabular}{lllll} 
CH O 262 & CH O & P4 & n/a & n/a \\
CH O 269 & CH O & P3 & n/a & n/a \\
H-GSP 81.14-4718 & Seh 81.14 & M2 & 1.63 & 2.25 \\
YGSP 40043 & Y680 (14.1) & P3 & 1.95 & 1.6 \\
\multicolumn{1}{c}{ Galerix rutlandae } & & & \\
CH BS 391 & CH BS & M2 & 1.78 & 2.42 \\
CH BS 392 & CH BS & M2 & 1.63 & 2.25 \\
CH BS 393 & CH BS & M1 & n/a & n/a \\
CH BS 401 & CH BS & P4 & 1.9 & n/a \\
CH BS 405 & CH BS & m1 & n/a & n/a \\
CH BS 410 & CH BS & M1 & n/a & n/a \\
CH BS 413 & CH BS & p3 & n/a & 0.97 \\
PMNH400x-1 & Dhok Tahlian & P4 & 2.07 & n/a \\
PMNH400x-2 & Dhok Tahlian & P4 & n/a & n/a \\
PMNH400x-3 & Dhok Tahlian & p1 & 1.15 & 0.47 \\
PMNH400x-4 & Dhok Tahlian & m1 & 2.4 & 1.73 \\
PMNH400x-5 & Dhok Tahlian & m3 & 1.58 & 1.12
\end{tabular}




\begin{tabular}{|c|c|c|c|c|}
\hline PMNH400x-7 & Dhok Tahlian & $\mathrm{m} 2$ & 1.93 & 1.58 \\
\hline PMNH400x-8 & Dhok Tahlian & $\mathrm{m} 2$ & 2 & 1.46 \\
\hline PMNH400x-9 & Dhok Tahlian & M3 & 0.99 & 1.68 \\
\hline H-GSP 82.14-4171 & Gaj 82.14 & M1 & $\mathrm{n} / \mathrm{a}$ & $\mathrm{n} / \mathrm{a}$ \\
\hline H-GSP 82.14-4174 & Gaj 82.14 & $\mathrm{p} 4$ & 1.2 & $\mathrm{n} / \mathrm{a}$ \\
\hline H-GSP 82.14-4175 & Gaj 82.14 & $\mathrm{p} 4$ & $\mathrm{n} / \mathrm{a}$ & $\mathrm{n} / \mathrm{a}$ \\
\hline H-GSP 82.14-4177 & Gaj 82.14 & $\mathrm{~m} 1 / 2$ & $\mathrm{n} / \mathrm{a}$ & $\mathrm{n} / \mathrm{a}$ \\
\hline H-GSP 82.24-1 & Seh 82.24 & $\mathrm{p} 4$ & 1.72 & 0.95 \\
\hline H-GSP 82.24-10 & Seh 82.24 & p3 & 1.65 & 0.88 \\
\hline H-GSP 82.24-18 & Seh 82.24 & $\mathrm{~m} 2$ & $\mathrm{n} / \mathrm{a}$ & 1.28 \\
\hline H-GSP 82.24-23 & Seh 82.24 & $\mathrm{~m} 3$ & 1.6 & 0.79 \\
\hline H-GSP 82.24-35 & Seh 82.24 & $\mathrm{P} 4$ & 1.88 & $\mathrm{n} / \mathrm{a}$ \\
\hline H-GSP 82.24-47 & Seh 82.24 & M2 & 1.8 & $\mathrm{n} / \mathrm{a}$ \\
\hline H-GSP 82.24-48 & Seh 82.24 & M2 & 1.85 & 2.47 \\
\hline H-GSP 82.24-53 & Seh 82.24 & M2 & 1.78 & 2.47 \\
\hline H-GSP 82.24-54 & Seh 82.24 & M2 & 1.6 & 2.44 \\
\hline H-GSP 82.24-55 & Seh 82.24 & M2 & 1.7 & 2.4 \\
\hline H-GSP 82.24-56 & Seh 82.24 & M2 & 1.56 & 1.93 \\
\hline H-GSP 82.24-59 & Seh 82.24 & M2 & 1.78 & 2.32 \\
\hline H-GSP 82.24-62 & Seh 82.24 & M3 & 1.09 & 1.51 \\
\hline H-GSP 82.24-64 & Seh 82.24 & M3 & 1.12 & 1.6 \\
\hline H-GSP 82.24-8 & Seh 82.24 & p3 & 1.6 & 0.82 \\
\hline UJZ29 & Seh 82.24 & $\mathrm{~m} 1$ & $\mathrm{n} / \mathrm{a}$ & $\mathrm{n} / \mathrm{a}$ \\
\hline UJZ34 & Seh 82.24 & $\mathrm{~m} 2$ & 2.05 & $\mathrm{n} / \mathrm{a}$ \\
\hline UJZ35 & Seh 82.24 & $\mathrm{~m} 1$ & $\mathrm{n} / \mathrm{a}$ & $\mathrm{n} / \mathrm{a}$ \\
\hline UJZ45 & Seh 82.24 & M2 & 1.73 & $\mathrm{n} / \mathrm{a}$ \\
\hline UJZ46 & Seh 82.24 & M2 & $\mathrm{n} / \mathrm{a}$ & $\mathrm{n} / \mathrm{a}$ \\
\hline UJZ47 & Seh 82.24 & M2 & $\mathrm{n} / \mathrm{a}$ & $\mathrm{n} / \mathrm{a}$ \\
\hline UJZ48 & Seh 82.24 & M2 & $\mathrm{n} / \mathrm{a}$ & $\mathrm{n} / \mathrm{a}$ \\
\hline UJZ52 & Seh 82.24 & M2 & $\mathrm{n} / \mathrm{a}$ & $\mathrm{n} / \mathrm{a}$ \\
\hline UJZ54 & Seh 82.24 & $\mathrm{~m} 1 / 2$ & $\mathrm{n} / \mathrm{a}$ & 1.38 \\
\hline UJZ69 & Seh 82.24 & $\mathrm{M} 1 / 2$ & $\mathrm{n} / \mathrm{a}$ & $\mathrm{n} / \mathrm{a}$ \\
\hline UJZ70 & Seh 82.24 & $\mathrm{P} 4$ & $\mathrm{n} / \mathrm{a}$ & $\mathrm{n} / \mathrm{a}$ \\
\hline YGSP 21832 & Y039 (13.6) & $\mathrm{p} 4$ & 2 & $\mathrm{n} / \mathrm{a}$ \\
\hline YGSP 24463 & Y430 (13.6) & $\mathrm{p} 1$ & 1.33 & 0.62 \\
\hline YGSP 24468 & Y430 (13.6) & p4 & 1.7 & 1.06 \\
\hline YGSP 24469 & Y430 (13.6) & $\mathrm{m} 3$ & 1.72 & 0.95 \\
\hline YGSP 24471 & Y430 (13.6) & $\mathrm{m} 2$ & 2.15 & 1.41 \\
\hline YGSP 24472 & Y430 (13.6) & $\mathrm{m} 2$ & 2 & 1.38 \\
\hline
\end{tabular}




\begin{tabular}{|c|c|c|c|c|c|}
\hline YGSP 39499 & \multicolumn{2}{|c|}{ Y430 (13.6) } & $\mathrm{P} 1$ & 1.19 & 0.52 \\
\hline YGSP 48412 & \multicolumn{2}{|c|}{ Y430 (13.6) } & p3 & 1.43 & 0.81 \\
\hline YGSP 17385 & \multicolumn{2}{|c|}{ Y491 (13.8) } & $\mathrm{P} 4$ & $\mathrm{n} / \mathrm{a}$ & $\mathrm{n} / \mathrm{a}$ \\
\hline YGSP 24337 & Y491 & 66 & $\mathrm{p} 4$ & 1.46 & $\mathrm{n} / \mathrm{a}$ \\
\hline YGSP 24429 & Y491 & “6 & $\mathrm{p} 4$ & 1.6 & $\mathrm{n} / \mathrm{a}$ \\
\hline YGSP 24433 & Y491 & “ & M1 & $\mathrm{n} / \mathrm{a}$ & $\mathrm{n} / \mathrm{a}$ \\
\hline YGSP 24434 & Y491 & 6 & $\mathrm{~m} 3$ & 1.68 & 0.93 \\
\hline YGSP 24440 & Y491 & 6 & M2 & $\mathrm{n} / \mathrm{a}$ & $\mathrm{n} / \mathrm{a}$ \\
\hline YGSP 24441 & Y491 & 66 & M2 & 1.8 & 2.42 \\
\hline YGSP 24442 & Y491 & 6 & M2 & $\mathrm{n} / \mathrm{a}$ & 1.95 \\
\hline YGSP 24443 & Y491 & 6 & M2 & $\mathrm{n} / \mathrm{a}$ & $\mathrm{n} / \mathrm{a}$ \\
\hline YGSP 24444 & Y491 & “6 & M2 & 1.63 & $\mathrm{n} / \mathrm{a}$ \\
\hline YGSP 24447 & Y491 & “ & $\mathrm{p} 4$ & 1.65 & $\mathrm{n} / \mathrm{a}$ \\
\hline YGSP 24449 & Y491 & 6 & $\mathrm{~m} 1$ & 2.4 & 1.51 \\
\hline YGSP 24450 & Y491 & 6 & $\mathrm{~m} 1$ & 2.49 & 1.75 \\
\hline YGSP 24451 & Y491 & “6 & $\mathrm{m} 3$ & 1.47 & 0.77 \\
\hline YGSP 24452 & Y491 & “6 & $\mathrm{p} 4$ & 1.78 & 1.26 \\
\hline YGSP 24453 & Y491 & 6 & M2 & 1.8 & 2.27 \\
\hline YGSP 24454 & Y491 & 6 & P3 & 1.51 & 1.26 \\
\hline YGSP 24455 & Y491 & 6 & M3 & 1.28 & 1.65 \\
\hline YGSP 24456 & Y491 & 6 & $\mathrm{~m} 1$ & 2.49 & 1.68 \\
\hline YGSP 24457 & Y491 & 66 & $\mathrm{~m} 2$ & 2.02 & 1.33 \\
\hline YGSP 24458 & Y491 & “ & $\mathrm{m} 3$ & 1.62 & 0.87 \\
\hline YGSP 24467 & Y491 & 66 & $\mathrm{p} 1$ & 1.28 & 0.64 \\
\hline YGSP 39473 & Y491 & “ & $\mathrm{m} 1$ & $\mathrm{n} / \mathrm{a}$ & $\mathrm{n} / \mathrm{a}$ \\
\hline YGSP 39478 & Y491 & “6 & $\mathrm{p} 1$ & 1.19 & 0.64 \\
\hline YGSP 40047 & Y491 & 6 & M3 & 1.16 & 1.58 \\
\hline YGSP 40048 & Y491 & “6 & M3 & 1.16 & 1.7 \\
\hline YGSP 49031 & Y491 & 6 & $\mathrm{~m} 3$ & $\mathrm{n} / \mathrm{a}$ & $\mathrm{n} / \mathrm{a}$ \\
\hline YGSP 49032 & Y491 & “ & $\mathrm{m} 2$ & $\mathrm{n} / \mathrm{a}$ & $\mathrm{n} / \mathrm{a}$ \\
\hline YGSP 49033 & Y491 & 6 & $\mathrm{~m} 1$ & $\mathrm{n} / \mathrm{a}$ & $\mathrm{n} / \mathrm{a}$ \\
\hline YGSP 49034 & Y491 & “ & M3 & 0.96 & 1.36 \\
\hline YGSP 49035 & Y491 & “6 & $\mathrm{m} 3$ & $\mathrm{n} / \mathrm{a}$ & 0.9 \\
\hline YGSP 49036 & Y491 & 66 & $\mathrm{~m} 1$ & $\mathrm{n} / \mathrm{a}$ & 1.48 \\
\hline YGSP 49037 & Y491 & “6 & $\mathrm{m} 1 / 2$ & $\mathrm{n} / \mathrm{a}$ & 1.58 \\
\hline YGSP 49038 & Y491 & 66 & $\mathrm{~m} 1 / 2$ & $\mathrm{n} / \mathrm{a}$ & 1.47 \\
\hline YGSP 49039 & Y491 & “ & $\mathrm{m} 1$ & $\mathrm{n} / \mathrm{a}$ & $\mathrm{n} / \mathrm{a}$ \\
\hline YGSP 49040 & Y491 & 6 & M2 & $\mathrm{n} / \mathrm{a}$ & $\mathrm{n} / \mathrm{a}$ \\
\hline YGSP 49041 & Y491 & “6 & $\mathrm{m} 3$ & $\mathrm{n} / \mathrm{a}$ & $\mathrm{n} / \mathrm{a}$ \\
\hline
\end{tabular}




\begin{tabular}{|c|c|c|c|c|}
\hline YGSP 49042 & Y491 (13.8) & $\mathrm{m} 3$ & $\mathrm{n} / \mathrm{a}$ & $\mathrm{n} / \mathrm{a}$ \\
\hline YGSP 49043 & Y491 (13.8) & $\mathrm{m} 1 / 2$ & $\mathrm{n} / \mathrm{a}$ & 1.55 \\
\hline YGSP 49044 & Y491 (13.8) & $\mathrm{m} 3$ & $\mathrm{n} / \mathrm{a}$ & $\mathrm{n} / \mathrm{a}$ \\
\hline YGSP 49045 & Y491 (13.8) & $\mathrm{m} 3$ & $\mathrm{n} / \mathrm{a}$ & $\mathrm{n} / \mathrm{a}$ \\
\hline YGSP 24498 & Y496 (12.3) & $\mathrm{m} 3$ & 1.7 & 0.94 \\
\hline YGSP 40013 & Y496 “ & M3 & 1.09 & 1.46 \\
\hline YGSP 40014 & Y496 “ & $\mathrm{p} 2$ & 1.19 & 0.62 \\
\hline YGSP 40015 & Y496 “ & $\mathrm{P} 4$ & $\mathrm{n} / \mathrm{a}$ & $\mathrm{n} / \mathrm{a}$ \\
\hline YGSP 40017 & Y496 “ & $\mathrm{C} 1$ & 1.19 & 0.52 \\
\hline YGSP 40018 & Y496 “ & M3 & 1.28 & 1.58 \\
\hline YGSP 40019 & Y496 “ & $\mathrm{p} 4$ & 1.43 & 0.96 \\
\hline YGSP 40020 & Y496 “ & $\mathrm{p} 2$ & 1.43 & 0.69 \\
\hline YGSP 40021 & Y496 “ & $\mathrm{P} 4$ & $\mathrm{n} / \mathrm{a}$ & $\mathrm{n} / \mathrm{a}$ \\
\hline YGSP 40022 & Y496 “ & $\mathrm{m} 3$ & 1.46 & 0.96 \\
\hline YGSP 40023 & Y496 “ & $\mathrm{P} 1$ & 1.26 & 0.54 \\
\hline YGSP 40024 & Y496 ، & $\mathrm{m} 2$ & 2.05 & 1.33 \\
\hline YGSP 40025 & Y496 “ & $\mathrm{p} 4$ & 1.6 & 1.06 \\
\hline YGSP 40026 & Y496 “ & $\mathrm{P} 2$ & 1.21 & 0.64 \\
\hline YGSP 40027 & Y496 “ & M2 & $\mathrm{n} / \mathrm{a}$ & $\mathrm{n} / \mathrm{a}$ \\
\hline YGSP 40028 & Y496 “ & p3 & 1.48 & 0.84 \\
\hline YGSP 40029 & Y496 “ & M2 & $\mathrm{n} / \mathrm{a}$ & 2.07 \\
\hline YGSP 40030 & Y496 “ & M2 & 1.73 & 2.35 \\
\hline YGSP 40031 & Y496 ، & M3 & 1.01 & 1.6 \\
\hline YGSP 40033 & Y496 “ & $\mathrm{p} 4$ & 1.63 & 1.01 \\
\hline YGSP 40034 & Y496 “ & $\mathrm{p} 2$ & 1.38 & 0.81 \\
\hline YGSP 40035 & Y496 “ & M2 & 1.83 & 2.38 \\
\hline YGSP 40036 & Y496 “ & $\mathrm{p} 1$ & 1.21 & 0.49 \\
\hline YGSP 24438 & Y504 (11.6) & $\mathrm{m} 3$ & 1.67 & 0.87 \\
\hline YGSP 24489 & Y504 (11.6) & $\mathrm{P} 3$ & 1.43 & 1.48 \\
\hline YGSP 39500 & Y504 (11.6) & $\mathrm{m} 2$ & $\mathrm{n} / \mathrm{a}$ & $\mathrm{n} / \mathrm{a}$ \\
\hline YGSP 24428 & Y589 (14.1) & $\mathrm{p} 4$ & 1.65 & $\mathrm{n} / \mathrm{a}$ \\
\hline YGSP 24431 & Y589 (14.1) & $\mathrm{m} 3$ & 1.68 & 0.89 \\
\hline YGSP 24432 & Y589 (14.1) & M1 & 2 & $\mathrm{n} / \mathrm{a}$ \\
\hline YGSP 39489 & Y589 (14.1) & $\mathrm{m} 3$ & $\mathrm{n} / \mathrm{a}$ & 0.9 \\
\hline YGSP 40037 & Y589 (14.1) & $\mathrm{P} 4$ & $\mathrm{n} / \mathrm{a}$ & $\mathrm{n} / \mathrm{a}$ \\
\hline YGSP 24435 & Y059 (13.6) & $\mathrm{P} 2$ & 1.21 & 0.59 \\
\hline YGSP 24436 & Y059 (13.6) & M3 & 1.16 & 1.6 \\
\hline YGSP 24445 & Y059 (13.6) & M2 & 1.78 & $\mathrm{n} / \mathrm{a}$ \\
\hline YGSP 24446 & Y059 (13.6) & M1 & $\mathrm{n} / \mathrm{a}$ & $\mathrm{n} / \mathrm{a}$ \\
\hline
\end{tabular}




\begin{tabular}{|c|c|c|c|c|}
\hline YGSP 24465 & Y059 (13.6) & $\mathrm{P} 4$ & 2.1 & 1.98 \\
\hline YGSP 24473 & Y059 (13.6) & M2 & 1.33 & 1.95 \\
\hline YGSP 24474 & Y059 (13.6) & $\mathrm{p} 2$ & $\mathrm{n} / \mathrm{a}$ & 0.79 \\
\hline YGSP 24476 & Y059 (13.6) & M3 & 1.11 & 1.58 \\
\hline YGSP 24477 & Y059 (13.6) & $\mathrm{m} 3$ & 1.57 & 0.85 \\
\hline YGSP 24495 & Y059 (13.6) & $\mathrm{p} 4$ & 1.43 & 1.06 \\
\hline YGSP 24503 & Y059 (13.6) & $\mathrm{p} 1$ & 1.14 & 0.57 \\
\hline YGSP 24510 & Y059 (13.6) & $\mathrm{C} 1$ & 1.14 & 0.52 \\
\hline YGSP 24513 & Y059 (13.6) & M2 & 1.53 & $\mathrm{n} / \mathrm{a}$ \\
\hline YGSP 39474 & Y059 (13.6) & M1 & $\mathrm{n} / \mathrm{a}$ & 2.64 \\
\hline YGSP 24460 & Y634 (12.3) & $\mathrm{P} 1$ & 1.11 & 0.64 \\
\hline YGSP 24478 & Y634 “ & D4 & 1.83 & 1.38 \\
\hline YGSP 24479 & Y634 “ & M2 & 1.95 & 2.25 \\
\hline YGSP 24480 & Y634 “ & P3 & 1.48 & 1.26 \\
\hline YGSP 24482 & Y634 “ & $\mathrm{m} 2$ & 2.05 & 1.36 \\
\hline YGSP 24484 & Y634 “ & M3 & 1.06 & 1.6 \\
\hline YGSP 24488 & Y634 “ & $\mathrm{m} 3$ & 1.43 & 0.82 \\
\hline YGSP 24496 & Y634 “ & $\mathrm{p} 2$ & 1.26 & 0.69 \\
\hline YGSP 24497 & Y634 “ & M1 & $\mathrm{n} / \mathrm{a}$ & $\mathrm{n} / \mathrm{a}$ \\
\hline YGSP 40039 & Y634 “ & $\mathrm{p} 4$ & 1.28 & $\mathrm{n} / \mathrm{a}$ \\
\hline YGSP 45189 & Y634 “ & M2 & $\mathrm{n} / \mathrm{a}$ & $\mathrm{n} / \mathrm{a}$ \\
\hline YGSP 24475 & Y640 (13.6) & $\mathrm{m} 2$ & 1.98 & 1.26 \\
\hline YGSP 33444 & Y640 “ & mandible & $\mathrm{n} / \mathrm{a}$ & $\mathrm{n} / \mathrm{a}$ \\
\hline YGSP 36282 & Y640 “ & M2 & $\mathrm{n} / \mathrm{a}$ & $\mathrm{n} / \mathrm{a}$ \\
\hline YGSP 36283 & Y640 “ & M2 & 1.76 & $\mathrm{n} / \mathrm{a}$ \\
\hline YGSP 36289 & Y640 “ & $\mathrm{m} 1$ & 2.58 & 1.5 \\
\hline YGSP 36290 & Y640 “ & M2 & 1.89 & 2.41 \\
\hline YGSP 36291 & Y640 “ & $\mathrm{m} 1$ & 2.52 & 1.6 \\
\hline YGSP 36292 & Y640 “ & M3 & 1.04 & 1.58 \\
\hline YGSP 39417 & Y640 “ & $\mathrm{m} 1$ & $\mathrm{n} / \mathrm{a}$ & 1.6 \\
\hline YGSP 40038 & Y640 “ & $\mathrm{m} 2$ & 2.1 & 1.6 \\
\hline YGSP 24427 & Y641 (13.7) & M2 & 1.8 & 2.17 \\
\hline YGSP 24509 & Y641 “ & $\mathrm{p} 4$ & 1.65 & $\mathrm{n} / \mathrm{a}$ \\
\hline YGSP 24511 & Y641 “ & P3 & 1.65 & 1.43 \\
\hline YGSP 24512 & Y641 “ & $\mathrm{p} 2$ & 1.28 & 0.74 \\
\hline YGSP 24515 & Y641 “ & P3 & $\mathrm{n} / \mathrm{a}$ & $\mathrm{n} / \mathrm{a}$ \\
\hline YGSP 24517 & Y641 ، & M1 & $\mathrm{n} / \mathrm{a}$ & $\mathrm{n} / \mathrm{a}$ \\
\hline YGSP 34848 & Y641 “" & $\mathrm{P} 3$ & 1.95 & 1.36 \\
\hline YGSP 34852 & Y641 “ & $\mathrm{P} 4$ & 2.1 & 1.65 \\
\hline
\end{tabular}




\begin{tabular}{|c|c|c|c|c|}
\hline YGSP 34853 & Y641 (13.7) & M1 & $\mathrm{n} / \mathrm{a}$ & $\mathrm{n} / \mathrm{a}$ \\
\hline YGSP 34854 & Y641 (13.7) & $\mathrm{m} 2$ & $\mathrm{n} / \mathrm{a}$ & $\mathrm{n} / \mathrm{a}$ \\
\hline YGSP 40041 & Y641 (13.7) & $\mathrm{m} 3$ & 1.67 & 0.95 \\
\hline YGSP 40044 & Y641 (13.7) & p3 & 1.53 & 0.79 \\
\hline YGSP 40045 & Y641 (13.7) & M2 & 1.5 & 2.48 \\
\hline YGSP 24437 & Y651 (13.6) & M1 & 2.02 & 2.3 \\
\hline YGSP 24464 & Y651 “ & $\begin{array}{l}\text { mandible } \\
\mathrm{m} 2-3\end{array}$ & $\mathrm{n} / \mathrm{a}$ & $\mathrm{n} / \mathrm{a}$ \\
\hline YGSP 24464a & Y651 “ & $\mathrm{m} 2$ & 1.98 & 1.58 \\
\hline YGSP 24470 & Y651 ، & M2 & $\mathrm{n} / \mathrm{a}$ & $\mathrm{n} / \mathrm{a}$ \\
\hline YGSP 24481 & Y651 “ & M2 & 1.58 & 2.07 \\
\hline YGSP 24493 & Y651 “ & M1 & 1.83 & 2.3 \\
\hline YGSP 24507 & Y651 “ & M2 & 1.78 & 2.32 \\
\hline YGSP 24461 & Y660 (13.7) & M2 & 1.78 & $\mathrm{n} / \mathrm{a}$ \\
\hline YGSP 24502 & Y660 (13.7) & M1 & $\mathrm{n} / \mathrm{a}$ & $\mathrm{n} / \mathrm{a}$ \\
\hline YGSP 34847 & Y665 (13.7) & $\mathrm{m} 2$ & $\mathrm{n} / \mathrm{a}$ & $\mathrm{n} / \mathrm{a}$ \\
\hline YGSP 24466 & Y668 (13.4) & D3 & 1.53 & 1.2 \\
\hline YGSP 39492 & Y668 (13.4) & $\mathrm{P} 4$ & 1.78 & $\mathrm{n} / \mathrm{a}$ \\
\hline YGSP 24508 & Y680 (14.1) & $\mathrm{m} 3$ & $\mathrm{n} / \mathrm{a}$ & 0.95 \\
\hline YGSP 24537 & Y680 (14.1) & M2 & 1.65 & 2.25 \\
\hline YGSP 24538 & Y680 (14.1) & M3 & 1.11 & 1.68 \\
\hline YGSP 34897 & Y690 (13.1) & p3 & 1.33 & 0.84 \\
\hline YGSP 34898 & Y690 (13.1) & M2 & 1.7 & 2.22 \\
\hline YGSP 34900 & Y690 (13.1) & $\mathrm{m} 3$ & 1.5 & 0.83 \\
\hline YGSP 34901 & Y690 (13.1) & $\mathrm{m} 2$ & 1.93 & 1.23 \\
\hline YGSP 34855 & Y698 (13.0) & $\mathrm{m} 1$ & 2.47 & 1.58 \\
\hline YGSP 34856 & Y698 (13.0) & $\mathrm{m} 1$ & 2.32 & 1.63 \\
\hline YGSP 34857 & Y698 (13.0) & $\mathrm{m} 2$ & 2 & 1.11 \\
\hline YGSP 34858 & Y698 (13.0) & $\mathrm{P} 4$ & 1.95 & 1.75 \\
\hline YGSP 34859 & Y698 (13.0) & M3 & 1.01 & 1.21 \\
\hline YGSP 49005 & Y698 (13.0) & M2 & $\mathrm{n} / \mathrm{a}$ & $\mathrm{n} / \mathrm{a}$ \\
\hline YGSP 49006 & Y698 (13.0) & $\mathrm{m} 3$ & $\mathrm{n} / \mathrm{a}$ & 0.9 \\
\hline YGSP 17386 & Y709 (14.3) & M2 & $\mathrm{n} / \mathrm{a}$ & 2.3 \\
\hline YGSP 17387 & Y709 “ & M1 & $\mathrm{n} / \mathrm{a}$ & $\mathrm{n} / \mathrm{a}$ \\
\hline YGSP 17388 & Y709 “ & P3 & 1.8 & 1.68 \\
\hline YGSP 24518 & Y709 “ & M2 & $\mathrm{n} / \mathrm{a}$ & $\mathrm{n} / \mathrm{a}$ \\
\hline YGSP 24519 & Y709 “ & p3 & 1.68 & 0.74 \\
\hline YGSP 24521 & Y709 “ & M1 & $\mathrm{n} / \mathrm{a}$ & $\mathrm{n} / \mathrm{a}$ \\
\hline YGSP 24522 & Y709 “ & M1 & 2.1 & 2.52 \\
\hline
\end{tabular}




\begin{tabular}{|c|c|c|c|c|c|}
\hline YGSP 24523 & Y709 & (14.3) & $\mathrm{p} 4$ & $\mathrm{n} / \mathrm{a}$ & $\mathrm{n} / \mathrm{a}$ \\
\hline YGSP 24524 & Y709 & “6 & $\mathrm{p} 4$ & 1.56 & 1.06 \\
\hline YGSP 24525 & Y709 & “ & M3 & 1.09 & 1.63 \\
\hline YGSP 24527 & Y709 & “6 & M1 & $\mathrm{n} / \mathrm{a}$ & $\mathrm{n} / \mathrm{a}$ \\
\hline YGSP 24528 & Y709 & “" & M1 & 1.93 & 2.25 \\
\hline YGSP 24529 & Y709 & “" & M2 & 1.68 & 2.35 \\
\hline YGSP 24531 & Y709 & “6 & M2 & 1.53 & 2.2 \\
\hline YGSP 24532 & Y709 & “6 & M3 & 1.04 & 1.63 \\
\hline YGSP 24534 & Y709 & “" & $\mathrm{p} 1$ & 1.11 & 0.52 \\
\hline YGSP 24535 & Y709 & “" & M2 & $\mathrm{n} / \mathrm{a}$ & $\mathrm{n} / \mathrm{a}$ \\
\hline YGSP 24536 & Y709 & “ & $\mathrm{p} 4$ & $\mathrm{n} / \mathrm{a}$ & $\mathrm{n} / \mathrm{a}$ \\
\hline YGSP 34850 & Y709 & “6 & M1 & $\mathrm{n} / \mathrm{a}$ & $\mathrm{n} / \mathrm{a}$ \\
\hline YGSP 40012 & Y709 & “" & M2 & $\mathrm{n} / \mathrm{a}$ & $\mathrm{n} / \mathrm{a}$ \\
\hline YGSP 21828 & Y714 & (12.8) & M1 & 2.15 & 2.12 \\
\hline YGSP 34865 & Y714 & “ & $\mathrm{m} 2$ & 2.02 & 1.36 \\
\hline YGSP 34866 & Y714 & “" & $\mathrm{p} 4$ & 1.51 & 0.99 \\
\hline YGSP 34867 & Y714 & “" & M3 & 0.94 & 1.21 \\
\hline YGSP 34868 & Y714 & “ & $\mathrm{m} 2$ & 2 & $\mathrm{n} / \mathrm{a}$ \\
\hline YGSP 34870 & Y714 & “ & $\mathrm{m} 2$ & 2 & 1.14 \\
\hline YGSP 34871 & Y714 & “" & P3 & 1.28 & $\mathrm{n} / \mathrm{a}$ \\
\hline YGSP 34872 & Y714 & “6 & M2 & 1.78 & 2.37 \\
\hline YGSP 34873 & Y714 & “6 & M3 & 1.11 & 1.41 \\
\hline YGSP 34874 & Y714 & “" & M2 & $\mathrm{n} / \mathrm{a}$ & $\mathrm{n} / \mathrm{a}$ \\
\hline YGSP 34875 & Y714 & “" & $\mathrm{C} 1$ & $\mathrm{n} / \mathrm{a}$ & 0.52 \\
\hline YGSP 34876 & Y714 & “" & $\mathrm{C} 1$ & 1.36 & 0.54 \\
\hline YGSP 34877 & Y714 & “6 & p3 & 1.33 & 0.91 \\
\hline YGSP 34878 & Y714 & “" & M2 & $\mathrm{n} / \mathrm{a}$ & $\mathrm{n} / \mathrm{a}$ \\
\hline YGSP 34879 & Y714 & “ & M2 & 1.78 & 2.3 \\
\hline YGSP 34882 & Y714 & “" & $\mathrm{P} 4$ & 2.05 & 2.1 \\
\hline YGSP 36187 & Y714 & “ & $\mathrm{m} 3$ & 1.62 & 0.83 \\
\hline YGSP 34902 & Y718 & (13.2) & $\mathrm{P} 1$ & 1.31 & 0.57 \\
\hline YGSP 34903 & Y718 & “" & M3 & 1.16 & 1.41 \\
\hline YGSP 34904 & Y718 & “6 & M3 & 0.99 & 1.6 \\
\hline YGSP 34905 & Y718 & “6 & $\mathrm{P} 4$ & $\mathrm{n} / \mathrm{a}$ & 1.8 \\
\hline YGSP 34906 & Y718 & “ & M3 & 1.09 & 1.41 \\
\hline YGSP 34907 & Y718 & “6 & M2 & 1.65 & 2.37 \\
\hline YGSP 34908 & Y718 & “ & $\mathrm{p} 4$ & 1.38 & 0.84 \\
\hline YGSP 34909 & Y718 & “" & $\mathrm{m} 2$ & $\mathrm{n} / \mathrm{a}$ & 1.16 \\
\hline YGSP 34910 & Y718 & “ & mandible & $\mathrm{n} / \mathrm{a}$ & $\mathrm{n} / \mathrm{a}$ \\
\hline
\end{tabular}




\begin{tabular}{|c|c|c|c|c|c|}
\hline & & & p3 & & \\
\hline YGSP 34910a & Y718 & 13.2) & p3 & 1.43 & 0.59 \\
\hline YGSP 34911 & Y718 & “6 & M2 & 1.63 & 2.12 \\
\hline YGSP 34912 & Y718 & 6 & M2 & 1.95 & 2.37 \\
\hline YGSP 34913 & Y718 & “6 & M2 & 1.83 & 2.42 \\
\hline YGSP 34914 & Y718 & “6 & $\mathrm{P} 2$ & 1.16 & 0.54 \\
\hline YGSP 34915 & Y718 & “6 & M3 & $\mathrm{n} / \mathrm{a}$ & 1.53 \\
\hline YGSP 34917 & Y718 & “6 & M1 & 2.07 & $\mathrm{n} / \mathrm{a}$ \\
\hline YGSP 34918 & Y718 & “ & $\mathrm{p} 4$ & 1.53 & 0.89 \\
\hline YGSP 34919 & Y718 & “6 & M1 & $\mathrm{n} / \mathrm{a}$ & 2.25 \\
\hline YGSP 34920 & Y718 & 6 & $\mathrm{~m} 2$ & $\mathrm{n} / \mathrm{a}$ & 1.46 \\
\hline YGSP 34922 & Y718 & “6 & M3 & $\mathrm{n} / \mathrm{a}$ & 1.41 \\
\hline YGSP 34923 & Y718 & “6 & $\mathrm{C} 1$ & 1.19 & 0.57 \\
\hline YGSP 34924 & Y718 & ، & M1 & $\mathrm{n} / \mathrm{a}$ & $\mathrm{n} / \mathrm{a}$ \\
\hline YGSP 34925 & Y718 & 6 & $\mathrm{~m} 1$ & 2.32 & 1.46 \\
\hline YGSP 34927 & Y718 & “6 & M3 & 0.96 & 1.6 \\
\hline YGSP 34929 & Y718 & “6 & M3 & 0.94 & 1.33 \\
\hline YGSP 34931 & Y718 & “6 & M3 & 0.91 & 1.53 \\
\hline YGSP 34932 & Y718 & 66 & M2 & 1.63 & 2.35 \\
\hline YGSP 34934 & Y718 & 6 & M2 & $\mathrm{n} / \mathrm{a}$ & $\mathrm{n} / \mathrm{a}$ \\
\hline YGSP 34935 & Y718 & “6 & $\mathrm{m} 3$ & 1.62 & 0.65 \\
\hline YGSP 36419 & Y718 & 6 & $\mathrm{~m} 2$ & 1.7 & $\mathrm{n} / \mathrm{a}$ \\
\hline YGSP 39452 & Y718 & “6 & $\mathrm{P} 4$ & $\mathrm{n} / \mathrm{a}$ & $\mathrm{n} / \mathrm{a}$ \\
\hline YGSP 40001 & Y718 & “ & $\mathrm{m} 2$ & 1.93 & 1.46 \\
\hline YGSP 40002 & Y718 & 66 & $\mathrm{p} 2$ & 1.14 & 0.77 \\
\hline YGSP 40003 & Y718 & 66 & $\mathrm{p} 4$ & 1.65 & 0.96 \\
\hline YGSP 40005 & Y718 & “6 & M3 & 1.19 & 1.36 \\
\hline YGSP 40006 & Y718 & “6 & $\mathrm{m} 1$ & 2.2 & 1.38 \\
\hline YGSP 40007 & Y718 & 6 & $\mathrm{p} 4$ & $\mathrm{n} / \mathrm{a}$ & $\mathrm{n} / \mathrm{a}$ \\
\hline YGSP 40009 & Y718 & “6 & M1 & $\mathrm{n} / \mathrm{a}$ & 2.31 \\
\hline YGSP 40010 & Y718 & 6 & M1 & 2.07 & 2.44 \\
\hline YGSP 40011 & Y718 & 66 & M1 & 2.17 & 2.49 \\
\hline YGSP 44440 & Y718 & “6 & M3 & 1.14 & $\mathrm{n} / \mathrm{a}$ \\
\hline YGSP 44441 & Y718 & 66 & M3 & 1.16 & 1.53 \\
\hline YGSP 44442 & Y718 & “6 & M3 & 0.94 & 1.43 \\
\hline YGSP 49021 & Y718 & “6 & M2 & $\mathrm{n} / \mathrm{a}$ & $\mathrm{n} / \mathrm{a}$ \\
\hline YGSP 49022 & Y718 & 6 & $\mathrm{p} 4$ & $\mathrm{n} / \mathrm{a}$ & 0.96 \\
\hline YGSP 49023 & Y718 & “ & $\mathrm{p} 4$ & 1.58 & $\mathrm{n} / \mathrm{a}$ \\
\hline YGSP 49024 & Y718 & “6 & $\mathrm{m} 3$ & $\mathrm{n} / \mathrm{a}$ & 0.75 \\
\hline
\end{tabular}




\begin{tabular}{|c|c|c|c|c|c|}
\hline YGSP 49025 & \multicolumn{2}{|c|}{ Y718 (13.2) } & $\mathrm{P} 4$ & $\mathrm{n} / \mathrm{a}$ & $\mathrm{n} / \mathrm{a}$ \\
\hline YGSP 49026 & \multicolumn{2}{|c|}{ Y718 (13.2) } & $\mathrm{m} 1 / 2$ & $\mathrm{n} / \mathrm{a}$ & 1.3 \\
\hline YGSP 49027 & \multicolumn{2}{|c|}{ Y718 (13.2) } & $\mathrm{m} 2$ & $\mathrm{n} / \mathrm{a}$ & $\mathrm{n} / \mathrm{a}$ \\
\hline YGSP 21830 & \multicolumn{2}{|c|}{ Y726 (13.0) } & M2 & $\mathrm{n} / \mathrm{a}$ & 2.25 \\
\hline YGSP 34861 & Y726 & “6 & $\begin{array}{l}\text { maxilla } \\
\text { M1-2 }\end{array}$ & $\mathrm{n} / \mathrm{a}$ & $\mathrm{n} / \mathrm{a}$ \\
\hline YGSP 34861a & Y726 & “ & M1 & 2.12 & 2.49 \\
\hline YGSP 34861b & Y726 & “ & M2 & 1.75 & 2.49 \\
\hline YGSP 34862 & Y726 & “ & mandible & $\mathrm{n} / \mathrm{a}$ & $\mathrm{n} / \mathrm{a}$ \\
\hline YGSP 34863 & Y726 & “ & $\begin{array}{l}\text { mandible } \\
\mathrm{m} 1-2\end{array}$ & $\mathrm{n} / \mathrm{a}$ & $\mathrm{n} / \mathrm{a}$ \\
\hline YGSP 34863a & Y726 & “ & $\mathrm{m} 1$ & 2.32 & 1.73 \\
\hline YGSP 34863b & Y726 & “ & $\mathrm{m} 2$ & 1.95 & 1.51 \\
\hline YGSP 34883 & Y726 & “ & maxilla P4 & $\mathrm{n} / \mathrm{a}$ & $\mathrm{n} / \mathrm{a}$ \\
\hline YGSP 34883a & Y726 & “ & $\mathrm{P} 4$ & 2.15 & 2.05 \\
\hline YGSP 34884 & Y726 & “ & mandible & $\mathrm{n} / \mathrm{a}$ & $\mathrm{n} / \mathrm{a}$ \\
\hline YGSP 34885 & Y726 & “ & maxilla P3 & $\mathrm{n} / \mathrm{a}$ & $\mathrm{n} / \mathrm{a}$ \\
\hline YGSP 34885a & Y726 & “ & $\mathrm{P} 3$ & 1.58 & 1.11 \\
\hline YGSP 34886 & Y726 & “ & $\mathrm{p} 1$ & 1.06 & 0.57 \\
\hline YGSP 34887 & Y726 & “ & M3 & $\mathrm{n} / \mathrm{a}$ & 1.7 \\
\hline YGSP 34888 & Y726 & “ & $\mathrm{m} 3$ & 1.6 & 0.83 \\
\hline YGSP 34889 & Y726 & “ & $\mathrm{m} 2$ & 2.22 & 1.36 \\
\hline YGSP 34891 & Y726 & “ & $\mathrm{p} 3$ & 1.43 & 0.74 \\
\hline YGSP 34892 & Y726 & “ & M3 & 1.16 & 1.46 \\
\hline YGSP 34893 & Y726 & “ & M2 & 1.85 & 2.37 \\
\hline YGSP 34894 & Y726 & “ & $\mathrm{m} 3$ & 1.55 & 0.97 \\
\hline YGSP 24539 & Y733 & 14.1) & $\mathrm{C} 1$ & 1.04 & 0.54 \\
\hline YGSP 24540 & Y733 & “ & M3 & 1.16 & 1.46 \\
\hline YGSP 24541 & Y733 & “6 & $\mathrm{p} 4$ & 1.68 & 1.01 \\
\hline YGSP 24543 & Y733 & “ & M3 & 0.94 & 1.46 \\
\hline YGSP 24545 & Y733 & “ & M3 & 1.26 & 1.68 \\
\hline YGSP 24546 & Y733 & “ & M3 & $\mathrm{n} / \mathrm{a}$ & 1.7 \\
\hline YGSP 24547 & Y733 & “ & M3 & 1.16 & 1.65 \\
\hline YGSP 24548 & Y733 & “ & $\mathrm{m} 1 / 2$ & $\mathrm{n} / \mathrm{a}$ & 1.35 \\
\hline YGSP 24549 & Y733 & “ & M3 & 1.14 & 1.6 \\
\hline YGSP 36188 & Y733 & “6 & M1 & $\mathrm{n} / \mathrm{a}$ & 2.49 \\
\hline YGSP 49013 & Y733 & “ & $\mathrm{m} 1$ & $\mathrm{n} / \mathrm{a}$ & 1.68 \\
\hline YGSP 49014 & Y733 & “ & M1 & 2.27 & $\mathrm{n} / \mathrm{a}$ \\
\hline YGSP 49015 & Y733 & “ & M3 & 1.19 & $\mathrm{n} / \mathrm{a}$ \\
\hline
\end{tabular}




\begin{tabular}{|c|c|c|c|c|}
\hline YGSP 49017 & Y733 (14.1) & $\mathrm{m} 1$ & $\mathrm{n} / \mathrm{a}$ & $\mathrm{n} / \mathrm{a}$ \\
\hline YGSP 17384 & Y734 (14.1) & M1 & 2.2 & 2.4 \\
\hline YGSP 48070 & Y750 (12.8) & $\mathrm{C} 1$ & 1.06 & 0.67 \\
\hline YGSP 48071 & Y750 (12.8) & $\mathrm{p} 1$ & 1.36 & 0.72 \\
\hline YGSP 48072 & Y750 “ & p3 & 1.31 & 0.69 \\
\hline YGSP 48073 & Y750 “ & p3 & 1.38 & 0.77 \\
\hline YGSP 48074 & Y750 “ & p3 & 1.48 & 0.77 \\
\hline YGSP 48075 & Y750 “ & p4 & 1.53 & 1.01 \\
\hline YGSP 48076 & Y750 “ & M2 & 1.76 & $\mathrm{n} / \mathrm{a}$ \\
\hline YGSP 48077 & Y750 “ & M2 & 1.76 & 2.41 \\
\hline YGSP 48078 & Y750 “ & M1 & 2.2 & 2.52 \\
\hline YGSP 48079 & Y750 “ & M2 & 1.73 & 2.17 \\
\hline YGSP 48080 & Y750 “ & M1 & $\mathrm{n} / \mathrm{a}$ & $\mathrm{n} / \mathrm{a}$ \\
\hline YGSP 48081 & Y750 “ & M2 & $\mathrm{n} / \mathrm{a}$ & $\mathrm{n} / \mathrm{a}$ \\
\hline YGSP 48082 & Y750 “ & M1 & $\mathrm{n} / \mathrm{a}$ & 2.25 \\
\hline YGSP 48083 & Y750 “ & M3 & 1.04 & 1.46 \\
\hline YGSP 48084 & Y750 “ & $\mathrm{m} 2$ & 2.05 & 1.33 \\
\hline YGSP 48085 & Y750 “ & $\mathrm{m} 2$ & 2.05 & 1.38 \\
\hline YGSP 48086 & Y750 “ & $\mathrm{P} 4$ & $\mathrm{n} / \mathrm{a}$ & $\mathrm{n} / \mathrm{a}$ \\
\hline YGSP 48087 & Y750 “ & $\mathrm{m} 1 / 2$ & $\mathrm{n} / \mathrm{a}$ & 1.68 \\
\hline YGSP 48088 & Y750 “ & $\mathrm{m} 1 / 2$ & $\mathrm{n} / \mathrm{a}$ & 1.43 \\
\hline YGSP 39466 & Y825 (12.7) & $\mathrm{m} 3$ & 1.52 & 0.8 \\
\hline YGSP 39467 & Y825 (12.7) & $\mathrm{m} 2$ & 1.9 & 1.46 \\
\hline YGSP 39468 & Y825 (12.7) & $\mathrm{m} 3$ & 1.6 & 0.97 \\
\hline YGSP 39469 & Y825 (12.7) & M1 & $\mathrm{n} / \mathrm{a}$ & $\mathrm{n} / \mathrm{a}$ \\
\hline YGSP 39480 & Y825 (12.7) & $\mathrm{P} 4$ & $\mathrm{n} / \mathrm{a}$ & $\mathrm{n} / \mathrm{a}$ \\
\hline YGSP 39481 & Y825 (12.7) & $\mathrm{P} 4$ & $\mathrm{n} / \mathrm{a}$ & $\mathrm{n} / \mathrm{a}$ \\
\hline YGSP 53937 & Y825 (12.7) & M2 & 1.75 & $\mathrm{n} / \mathrm{a}$ \\
\hline YGSP 53938 & Y825 (12.7) & p3 & 1.36 & 0.79 \\
\hline YGSP 53939 & Y825 (12.7) & $\mathrm{p} 4$ & 1.65 & 1.11 \\
\hline YGSP 53940 & Y825 (12.7) & $\mathrm{m} 1$ & 2.42 & 1.36 \\
\hline YGSP 53941 & Y825 (12.7) & $\mathrm{m} 2$ & $\mathrm{n} / \mathrm{a}$ & 1.48 \\
\hline YGSP 53942 & Y825 (12.7) & $\mathrm{m} 1 / 2$ & $\mathrm{n} / \mathrm{a}$ & 1.3 \\
\hline YGSP 53943 & Y825 (12.7) & $\mathrm{m} 1$ & 2.49 & 1.6 \\
\hline YGSP 53944 & Y825 (12.7) & $\mathrm{m} 1$ & 2.2 & 1.56 \\
\hline YGSP 53946 & Y825 (12.7) & $\mathrm{P} 4$ & $\mathrm{n} / \mathrm{a}$ & $\mathrm{n} / \mathrm{a}$ \\
\hline YGSP 53950 & Y825 (12.7) & $\mathrm{P} 1$ & 0.94 & 0.54 \\
\hline
\end{tabular}

Galerix sp.

$\begin{array}{lllll}\text { H-GSP 82.24-17 } & \text { Seh 82.24 } 2.07 \quad \text { n/a }\end{array}$ 


\begin{tabular}{|c|c|c|c|c|}
\hline H-GSP 82.24-22 & Seh 82.24 & $\mathrm{~m} 3$ & 1.53 & 1.04 \\
\hline H-GSP 82.24-38 & Seh 82.24 & $\mathrm{P} 4$ & $\mathrm{n} / \mathrm{a}$ & 2.15 \\
\hline H-GSP 82.24-42 & Seh 82.24 & $\mathrm{P} 4$ & 2.15 & $\mathrm{n} / \mathrm{a}$ \\
\hline H-GSP 82.24-51 & Seh 82.24 & M2 & 1.9 & 2.57 \\
\hline H-GSP 82.24-52 & Seh 82.24 & M2 & $\mathrm{n} / \mathrm{a}$ & $\mathrm{n} / \mathrm{a}$ \\
\hline UJZ23 & Seh 82.24 & $\mathrm{~m} 3$ & $\mathrm{n} / \mathrm{a}$ & $\mathrm{n} / \mathrm{a}$ \\
\hline UJZ25 & Seh 82.24 & $\mathrm{~m} 2$ & $\mathrm{n} / \mathrm{a}$ & $\mathrm{n} / \mathrm{a}$ \\
\hline UJZ26 & Seh 82.24 & $\mathrm{~m} 3$ & $\mathrm{n} / \mathrm{a}$ & $\mathrm{n} / \mathrm{a}$ \\
\hline UJZ28 & Seh 82.24 & $\mathrm{~m} 2$ & $\mathrm{n} / \mathrm{a}$ & $\mathrm{n} / \mathrm{a}$ \\
\hline UJZ31 & Seh 82.24 & $\mathrm{~m} 3$ & $\mathrm{n} / \mathrm{a}$ & $\mathrm{n} / \mathrm{a}$ \\
\hline UJZ32 & Seh 82.24 & $\mathrm{~m} 2$ & $\mathrm{n} / \mathrm{a}$ & $\mathrm{n} / \mathrm{a}$ \\
\hline UJZ33 & Seh 82.24 & $\mathrm{~m} 1$ & $\mathrm{n} / \mathrm{a}$ & $\mathrm{n} / \mathrm{a}$ \\
\hline UJZ43 & Seh 82.24 & $\mathrm{~m} 1$ & $\mathrm{n} / \mathrm{a}$ & 1.63 \\
\hline UJZ50 & Seh 82.24 & M1 & $\mathrm{n} / \mathrm{a}$ & $\mathrm{n} / \mathrm{a}$ \\
\hline UJZ51 & Seh 82.24 & $\mathrm{~m} 1 / 2$ & $\mathrm{n} / \mathrm{a}$ & 1.53 \\
\hline UJZ59 & Seh 82.24 & $\mathrm{~m} 1 / 2$ & $\mathrm{n} / \mathrm{a}$ & 1.68 \\
\hline UJZ62 & Seh 82.24 & $\mathrm{P} 4$ & $\mathrm{n} / \mathrm{a}$ & $\mathrm{n} / \mathrm{a}$ \\
\hline UJZ64 & Seh 82.24 & $\mathrm{P} 4$ & $\mathrm{n} / \mathrm{a}$ & $\mathrm{n} / \mathrm{a}$ \\
\hline UJZ68 & Seh 82.24 & $\mathrm{p} 4$ & $\mathrm{n} / \mathrm{a}$ & $\mathrm{n} / \mathrm{a}$ \\
\hline YGSP 34845 & Y709 (14.3) & $\mathrm{P} 1$ & 1.33 & 0.54 \\
\hline YGSP 34849 & Y709 (14.3) & $\mathrm{P} 2$ & 1.38 & 0.62 \\
\hline YGSP 39479 & Y709 (14.3) & M2 & 1.8 & 2.25 \\
\hline YGSP 49050 & Y709 (14.3) & $\mathrm{p} 4$ & $\mathrm{n} / \mathrm{a}$ & $\mathrm{n} / \mathrm{a}$ \\
\hline YGSP 24462 & Y733 (14.1) & $\mathrm{P} 1$ & $\mathrm{n} / \mathrm{a}$ & 0.44 \\
\hline YGSP 24544 & Y733 (14.1) & $\mathrm{m} 3$ & 1.65 & 0.89 \\
\hline YGSP 49016 & Y733 (14.1) & $\mathrm{m} 2$ & $\mathrm{n} / \mathrm{a}$ & $\mathrm{n} / \mathrm{a}$ \\
\hline \multicolumn{5}{|c|}{ Galerix wesselsae } \\
\hline UJZ1 & CH BS 1978 & $\mathrm{P} 4$ & $\mathrm{n} / \mathrm{a}$ & $\mathrm{n} / \mathrm{a}$ \\
\hline UJZ2 & CH BS 1978 & $\mathrm{P} 4$ & $\mathrm{n} / \mathrm{a}$ & $\mathrm{n} / \mathrm{a}$ \\
\hline CH O 268 & $\mathrm{CHO}$ & M3 & 1.33 & 1.7 \\
\hline CH O 270 & $\mathrm{CHO}$ & $\mathrm{m} 1$ & $\mathrm{n} / \mathrm{a}$ & $\mathrm{n} / \mathrm{a}$ \\
\hline H-GSP 81.14-4703 & Seh 81.14 & p3 & 1.43 & 0.74 \\
\hline H-GSP 81.14-4704 & Seh 81.14 & $\mathrm{P} 2$ & $\mathrm{n} / \mathrm{a}$ & $\mathrm{n} / \mathrm{a}$ \\
\hline H-GSP 81.14-4707 & Seh 81.14 & $\mathrm{p} 4$ & 1.8 & 1.23 \\
\hline H-GSP 81.14-4710 & Seh 81.14 & $\mathrm{~m} 2$ & 2.4 & $\mathrm{n} / \mathrm{a}$ \\
\hline H-GSP 81.14-4713 & Seh 81.14 & $\mathrm{P} 4$ & $\mathrm{n} / \mathrm{a}$ & 2.17 \\
\hline H-GSP 81.14-4715 & Seh 81.14 & M2 & 2.15 & 2.87 \\
\hline H-GSP 81.14-4716 & Seh 81.14 & M2 & 2.15 & 2.67 \\
\hline H-GSP 81.14-4717 & Seh 81.14 & M1 & $\mathrm{n} / \mathrm{a}$ & $\mathrm{n} / \mathrm{a}$ \\
\hline
\end{tabular}




\begin{tabular}{|c|c|c|c|c|}
\hline H-GSP 81.14-4720 & Seh 81.14 & M3 & 1.28 & 1.68 \\
\hline UJZ14 & Seh 81.14 & $\mathrm{P} 4$ & $\mathrm{n} / \mathrm{a}$ & $\mathrm{n} / \mathrm{a}$ \\
\hline UJZ16 & Seh 81.14 & $\mathrm{P} 4$ & $\mathrm{n} / \mathrm{a}$ & $\mathrm{n} / \mathrm{a}$ \\
\hline UJZ19 & Seh 81.14 & $\mathrm{~m} 1 / 2$ & $\mathrm{n} / \mathrm{a}$ & 1.83 \\
\hline H-GSP 81.14a-4282 & Seh $81.14 \mathrm{a}$ & $\mathrm{p} 2$ & 1.38 & 1.04 \\
\hline H-GSP 81.14a-4283 & Seh $81.14 \mathrm{a}$ & $\mathrm{p} 4$ & 1.8 & 1.16 \\
\hline H-GSP 81.14a-4284 & Seh $81.14 a$ & $\mathrm{~m} 1$ & $\mathrm{n} / \mathrm{a}$ & $\mathrm{n} / \mathrm{a}$ \\
\hline H-GSP 81.14a-4287 & Seh $81.14 \mathrm{a}$ & $\mathrm{m} 2$ & $\mathrm{n} / \mathrm{a}$ & $\mathrm{n} / \mathrm{a}$ \\
\hline H-GSP 81.14a-4290 & Seh $81.14 \mathrm{a}$ & $\mathrm{m} 3$ & $\mathrm{n} / \mathrm{a}$ & $\mathrm{n} / \mathrm{a}$ \\
\hline H-GSP 81.14a-4291 & Seh $81.14 \mathrm{a}$ & $\mathrm{P} 4$ & 2.2 & $\mathrm{n} / \mathrm{a}$ \\
\hline H-GSP 81.14a-4294 & Seh $81.14 \mathrm{a}$ & $\mathrm{m} 1 / 2$ & $\mathrm{n} / \mathrm{a}$ & 1.85 \\
\hline H-GSP 81.14a-4296 & Seh $81.14 \mathrm{a}$ & $\mathrm{P} 4$ & $\mathrm{n} / \mathrm{a}$ & $\mathrm{n} / \mathrm{a}$ \\
\hline H-GSP 81.14a-4297 & Seh $81.14 \mathrm{a}$ & $\mathrm{m} 2$ & $\mathrm{n} / \mathrm{a}$ & 1.57 \\
\hline H-GSP 81.14a-4300 & Seh $81.14 \mathrm{a}$ & $\mathrm{m} 3$ & $\mathrm{n} / \mathrm{a}$ & $\mathrm{n} / \mathrm{a}$ \\
\hline H-GSP 81.14a-4303 & Seh $81.14 \mathrm{a}$ & $\mathrm{m} 2$ & 2.22 & 1.51 \\
\hline H-GSP 81.14a-4304 & Seh $81.14 \mathrm{a}$ & M3 & 1.23 & 2.02 \\
\hline H-GSP 81.14a-4305 & Seh $81.14 \mathrm{a}$ & $\mathrm{P} 2$ & 1.26 & 0.72 \\
\hline H-GSP 81.14a-4306 & Seh $81.14 \mathrm{a}$ & $\mathrm{P} 3$ & 1.88 & $\mathrm{n} / \mathrm{a}$ \\
\hline H-GSP 81.14a-4307 & Seh $81.14 \mathrm{a}$ & M2 & 2.02 & 2.58 \\
\hline H-GSP 81.14a-4308 & Seh $81.14 \mathrm{a}$ & M2 & 2 & $\mathrm{n} / \mathrm{a}$ \\
\hline H-GSP 81.14a-4309 & Seh $81.14 \mathrm{a}$ & P3 & 1.73 & 1.95 \\
\hline H-GSP 81.14a-4310 & Seh $81.14 \mathrm{a}$ & M2 & 1.9 & 2.44 \\
\hline H-GSP 82.24-11 & Seh 82.24 & $\mathrm{p} 4$ & 1.83 & 1.21 \\
\hline H-GSP 82.24-12 & Seh 82.24 & p3 & 1.63 & 0.96 \\
\hline H-GSP 82.24-14 & Seh 82.24 & $\mathrm{~m} 1$ & 2.44 & 1.7 \\
\hline H-GSP 82.24-16 & Seh 82.24 & $\mathrm{~m} 2$ & 2.25 & $\mathrm{n} / \mathrm{a}$ \\
\hline H-GSP 82.24-21 & Seh 82.24 & $\mathrm{~m} 3$ & 1.7 & 1.06 \\
\hline H-GSP 82.24-34 & Seh 82.24 & P3 & 2 & 1.68 \\
\hline H-GSP 82.24-39 & Seh 82.24 & $\mathrm{P} 4$ & 2.25 & 2.25 \\
\hline H-GSP 82.24-40 & Seh 82.24 & $\mathrm{P} 4$ & $\mathrm{n} / \mathrm{a}$ & 2.32 \\
\hline H-GSP 82.24-6 & Seh 82.24 & $\mathrm{p} 1$ & 1.56 & 0.64 \\
\hline H-GSP 82.24-61 & Seh 82.24 & M3 & 1.21 & 1.6 \\
\hline H-GSP 82.24-63 & Seh 82.24 & M3 & 1.09 & 1.83 \\
\hline H-GSP 82.24-66 & Seh 82.24 & M3 & 1.21 & 1.7 \\
\hline H-GSP 82.24-73 & Seh 82.24 & $\mathrm{p} 4$ & $\mathrm{n} / \mathrm{a}$ & 1.23 \\
\hline UJZ36 & Seh 82.24 & $\mathrm{~m} 1$ & $\mathrm{n} / \mathrm{a}$ & $\mathrm{n} / \mathrm{a}$ \\
\hline UJZ55 & Seh 82.24 & $\mathrm{p} 4$ & 1.68 & 1.19 \\
\hline H-GSP 82.27-4411 & Seh 82.27 & M3 & 1.33 & 1.78 \\
\hline H-GSP 82.27-4421 & Seh 82.27 & $\mathrm{~m} 2$ & 2.44 & $\mathrm{n} / \mathrm{a}$ \\
\hline
\end{tabular}




\begin{tabular}{|c|c|c|c|c|}
\hline H-GSP 82.27-4426 & Seh 82.27 & M1 & $\mathrm{n} / \mathrm{a}$ & $\mathrm{n} / \mathrm{a}$ \\
\hline H-GSP 84.24-4241 & Seh 84.24 & P3 & 1.51 & 1.95 \\
\hline H-GSP 84.24-4242 & Seh 84.24 & $\mathrm{P} 4$ & 2.3 & $\mathrm{n} / \mathrm{a}$ \\
\hline H-GSP 84.24-4244 & Seh 84.24 & $\mathrm{P} 2$ & 1.14 & 0.72 \\
\hline H-GSP 84.24-4247 & Seh 84.24 & M1 & $\mathrm{n} / \mathrm{a}$ & $\mathrm{n} / \mathrm{a}$ \\
\hline H-GSP 84.24-4248 & Seh 84.24 & $\mathrm{~m} 3$ & 1.78 & 0.91 \\
\hline H-GSP 84.24-4250 & Seh 84.24 & M2 & $\mathrm{n} / \mathrm{a}$ & 1.99 \\
\hline H-GSP 84.24-4253 & Seh 84.24 & $\mathrm{P} 1$ & 0.99 & 0.47 \\
\hline H-GSP 84.24-4254 & Seh 84.24 & $\mathrm{p} 4$ & 1.63 & $\mathrm{n} / \mathrm{a}$ \\
\hline H-GSP 84.24-4256 & Seh 84.24 & $\mathrm{p} 2$ & 1.53 & 0.78 \\
\hline H-GSP 84.24-4257 & Seh 84.24 & $\mathrm{p} 1$ & 1.33 & 0.62 \\
\hline H-GSP 84.24-4258 & Seh 84.24 & $\mathrm{~m} 2$ & $\mathrm{n} / \mathrm{a}$ & 1.75 \\
\hline H-GSP 84.25-4151 & Seh 84.25 & M2 & 1.92 & 2.58 \\
\hline H-GSP 84.25-4152 & Seh 84.25 & $\mathrm{~m} 1$ & $\mathrm{n} / \mathrm{a}$ & $\mathrm{n} / \mathrm{a}$ \\
\hline H-GSP 84.26-4174 & Seh 84.26 & $\mathrm{~m} 3$ & 1.73 & 1.11 \\
\hline H-GSP 84.26-4176 & Seh 84.26 & M3 & 1.21 & 1.95 \\
\hline H-GSP 84.26-4177 & Seh 84.26 & $\mathrm{~m} 1 / 2$ & $\mathrm{n} / \mathrm{a}$ & 1.68 \\
\hline H-GSP 84.26-4180 & Seh 84.26 & $\mathrm{~m} 2$ & $\mathrm{n} / \mathrm{a}$ & $\mathrm{n} / \mathrm{a}$ \\
\hline H-GSP 84.26-4182 & Seh 84.26 & $\mathrm{~m} 1 / 2$ & $\mathrm{n} / \mathrm{a}$ & 1.65 \\
\hline H-GSP 84.26-4183 & Seh 84.26 & $\mathrm{p} 4$ & $\mathrm{n} / \mathrm{a}$ & $\mathrm{n} / \mathrm{a}$ \\
\hline H-GSP 84.26-4184 & Seh 84.26 & P3 & 1.48 & 1.78 \\
\hline YGSP 24500 & Y592 (16.0) & $\mathrm{p} 4$ & 1.83 & $\mathrm{n} / \mathrm{a}$ \\
\hline YGSP 24504 & Y592 (16.0) & $\mathrm{P} 4$ & $\mathrm{n} / \mathrm{a}$ & $\mathrm{n} / \mathrm{a}$ \\
\hline YGSP 40042 & Y592 (16.0) & $\mathrm{m} 1 / 2$ & $\mathrm{n} / \mathrm{a}$ & 1.68 \\
\hline YGSP 49002 & Y592 (16.0) & P3 & 1.6 & $\mathrm{n} / \mathrm{a}$ \\
\hline YGSP 49012 & Y642 (15.2) & $\mathrm{P} 4$ & 2.02 & $\mathrm{n} / \mathrm{a}$ \\
\hline YGSP 52202 & Y642 (15.2) & M1 & 2.58 & 2.64 \\
\hline YGSP 52203 & Y642 (15.2) & $\mathrm{P} 1$ & 0.84 & 0.57 \\
\hline YGSP 48061 & Y678 (15.1) & $\mathrm{P} 2$ & $\mathrm{n} / \mathrm{a}$ & 0.62 \\
\hline YGSP 36189 & Y682 (15.2) & M3 & 1.16 & 1.63 \\
\hline YGSP 36190 & Y682 (15.2) & M1 & $\mathrm{n} / \mathrm{a}$ & $\mathrm{n} / \mathrm{a}$ \\
\hline YGSP 39472 & Y682 (15.2) & $\mathrm{m} 1$ & $\mathrm{n} / \mathrm{a}$ & 1.63 \\
\hline YGSP 48031 & Y682 (15.2) & M3 & 1.14 & 1.65 \\
\hline YGSP 39491 & Y692 (16-17) & $\mathrm{m} 1 / 2$ & $\mathrm{n} / \mathrm{a}$ & 1.53 \\
\hline YGSP 24530 & Y709 (14.3) & $\mathrm{P} 4$ & 1.75 & 2.05 \\
\hline YGSP 24533 & Y709 (14.3) & M2 & 1.83 & 2.67 \\
\hline YGSP 34851 & Y709 (14.3) & $\mathrm{m} 1$ & 2.67 & 1.5 \\
\hline YGSP 40046 & Y709 (14.3) & M2 & $\mathrm{n} / \mathrm{a}$ & $\mathrm{n} / \mathrm{a}$ \\
\hline
\end{tabular}




\begin{tabular}{|c|c|c|c|c|}
\hline YGSP 49051 & Y721 (17.8) & $\mathrm{p} 4$ & $\mathrm{n} / \mathrm{a}$ & 1.2 \\
\hline YGSP 52205 & Y721 (17.8) & M3 & 1.33 & $\mathrm{n} / \mathrm{a}$ \\
\hline YGSP 24542 & Y733 (14.1) & $\mathrm{m} 1$ & 2.67 & 1.7 \\
\hline YGSP 24515 & Y747 (17.8) & M2 & $\mathrm{n} / \mathrm{a}$ & $\mathrm{n} / \mathrm{a}$ \\
\hline YGSP 39470 & Y747 ، & $\mathrm{m} 2$ & 2.2 & $\mathrm{n} / \mathrm{a}$ \\
\hline YGSP 39490 & Y747 “ & $\mathrm{m} 1 / 2$ & $\mathrm{n} / \mathrm{a}$ & 1.57 \\
\hline YGSP 48063 & Y747 “ & $\mathrm{C} 1$ & 1.52 & 0.78 \\
\hline YGSP 48066 & Y747 “ & M2 & 1.89 & 2.67 \\
\hline YGSP 48067 & Y747 “ & M1 & $\mathrm{n} / \mathrm{a}$ & $\mathrm{n} / \mathrm{a}$ \\
\hline YGSP 36191 & Y802 (16.8) & $\mathrm{P} 4$ & 2.61 & $\mathrm{n} / \mathrm{a}$ \\
\hline YGSP 36192 & Y802 “ & $\mathrm{P} 4$ & 2.3 & $\mathrm{n} / \mathrm{a}$ \\
\hline YGSP 36193 & Y802 “ & $\mathrm{P} 4$ & 2.2 & $\mathrm{n} / \mathrm{a}$ \\
\hline YGSP 36194 & Y802 “ & $\mathrm{P} 4$ & $\mathrm{n} / \mathrm{a}$ & $\mathrm{n} / \mathrm{a}$ \\
\hline YGSP 39471 & Y802 “ & $\mathrm{p} 2$ & 1.53 & 0.81 \\
\hline YGSP 48045 & Y802 “ & $\mathrm{C} 1$ & 1.38 & $\mathrm{n} / \mathrm{a}$ \\
\hline YGSP 48046 & Y802 “ & p3 & 1.25 & 0.7 \\
\hline Z 2297 & Z122 (18.9) & $\mathrm{p} 2$ & 1.37 & 0.8 \\
\hline Z 2649 & Z122 “ & $\mathrm{p} 4$ & 1.65 & $\mathrm{n} / \mathrm{a}$ \\
\hline Z 568 & Z122 “ & M3 & 1.21 & 1.9 \\
\hline Z 569 & Z122 “ & $\mathrm{P} 2$ & 1.36 & 0.67 \\
\hline Z 571 & Z122 “ & M1 & $\mathrm{n} / \mathrm{a}$ & $\mathrm{n} / \mathrm{a}$ \\
\hline Z 572 & $\mathrm{Z} 122$ & $\begin{array}{l}\text { mandible } \\
\mathrm{m} 2\end{array}$ & $\mathrm{n} / \mathrm{a}$ & $\mathrm{n} / \mathrm{a}$ \\
\hline Z 572a & Z122 “ & $\mathrm{m} 2$ & 2.3 & 1.51 \\
\hline Z 573 & Z122 “ & M2 & 1.65 & 2.52 \\
\hline Z 574 & Z122 “ & $\mathrm{m} 1$ & $\mathrm{n} / \mathrm{a}$ & $\mathrm{n} / \mathrm{a}$ \\
\hline Z 575 & Z122 “ & $\mathrm{m} 2$ & $\mathrm{n} / \mathrm{a}$ & 1.45 \\
\hline YGSP 21835 & Z124 (19.0) & M1 & $\mathrm{n} / \mathrm{a}$ & $\mathrm{n} / \mathrm{a}$ \\
\hline YGSP 21836 & $\mathrm{Z} 124$ & $\mathrm{p} 4$ & 1.98 & $\mathrm{n} / \mathrm{a}$ \\
\hline YGSP 21837 & $\mathrm{Z} 124$ & P3 & 2 & $\mathrm{n} / \mathrm{a}$ \\
\hline YGSP 39488 & Z124 “ & $\mathrm{m} 1 / 2$ & $\mathrm{n} / \mathrm{a}$ & 1.4 \\
\hline Z 617 & Z124 “ & M2 & $\mathrm{n} / \mathrm{a}$ & $\mathrm{n} / \mathrm{a}$ \\
\hline Z 2630 & Z167 (18.9) & M1 & 2.44 & $\mathrm{n} / \mathrm{a}$ \\
\hline \multicolumn{5}{|c|}{ Schizogalerix sp. } \\
\hline YGSP 17255 & Y259 (10.5) & $\mathrm{P} 3 / 4$ & $\mathrm{n} / \mathrm{a}$ & $\mathrm{n} / \mathrm{a}$ \\
\hline YGSP 24490 & Y259 (10.5) & $\mathrm{p} 4$ & 1.85 & 1.16 \\
\hline YGSP 24491 & Y259 (10.5) & p3 & 1.43 & 0.86 \\
\hline YGSP 24492 & Y259 (10.5) & M2 & 1.99 & 2.77 \\
\hline
\end{tabular}




$\begin{array}{lllll}\text { YGSP 39486 } & \text { Y259 (10.5) } & \text { P2 } & 1.28 & 0.72 \\ \text { YGSP 39487 } & \text { Y259 (10.5) } & \text { m1/2 } & \text { n/a } & 1.43 \\ \text { YGSP 39496 } & \text { Y259 (10.5) } & \text { M1/2 } & \text { n/a } & \text { n/a } \\ \text { YGSP 49003 } & \text { Y259 (10.5) } & \text { P3 } & 2.12 & \text { n/a } \\ \text { YGSP 49004 } & \text { Y259 (10.5) } & \text { P4 } & 2.61 & \text { n/a } \\ \text { YGSP 24459 } & \text { Y450(10.2) } & \text { P2 } & 1.53 & 0.86 \\ \text { YGSP 21833 } & \text { Y076 (11.4) } & \text { D3 } & 1.72 & 1.05 \\ \text { YGSP 34946 } & \text { Y791 (11.2) } & \text { P4 } & 2.2 & \text { n/a }\end{array}$

